"Mircea cel Batran" Naval Academy Scientific Bulletin, Volume XX - 2017 - Issue 2

Published by "Mircea cel Batran" Naval Academy Press, Romania // The journal is indexed in: PROQUEST I DOAJ / DRJI / JOURNAL INDEX / I2OR / SCIENCE LIBRARY INDEX / Google Scholar / Crossref / Academic Keys I ROAD Open Access / OAJI / Academic Resources / Scientific Indexing Services / SCIPIO / JIFACTOR

\title{
CFD RESULTS ON PROPELLER ANALYSIS
}

\section{Adrian POPA, Academia Navală Mircea cel Bătrân Constanța România Ionuț Cristian SCURTU, Academia Navală Mircea cel Bătrân Constanța România}

Abstract: The Ansys software will be used to determine the pressures and velocity generated by the propeller with 5 blades in lateral currents. The CFX component used will generate the characteristic velocity, pressure and turbulence ratios for the studied fluid field with a very precise mesh analysis. All data presented from simulation results can be used for shaft dimensioning and further analysis.

Keywords: propeller, CFD, analysis, software Ansys

\section{Introduction}

Ansys Workbench work process for CFD simulation is presented below with operations included:

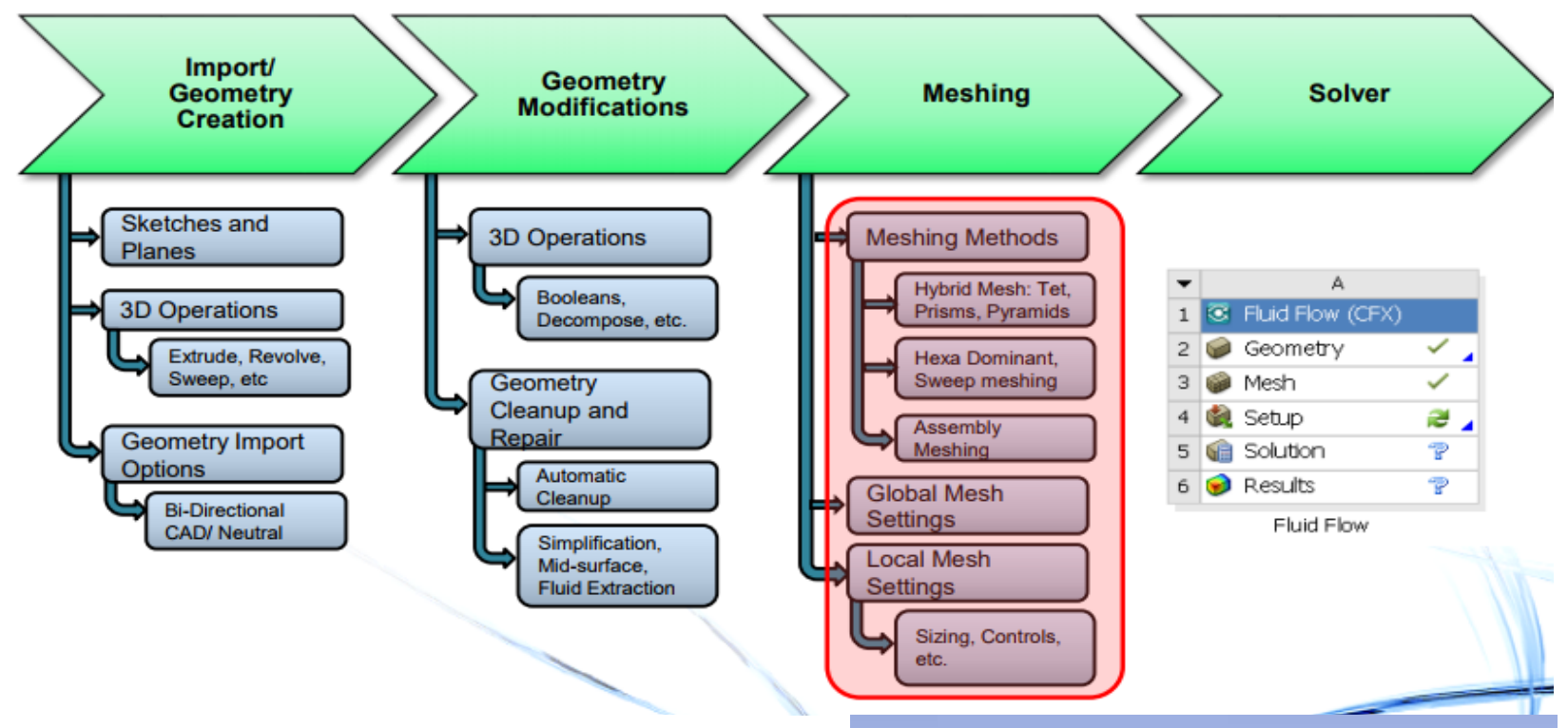

Problem description and introduction Ansys Workbench modeling is presented in Geomery design Modeler for a 5 bladed propeller used in lateral CFD flow tests. We will use a 5 blades propeller importd as STL file in Ansys.

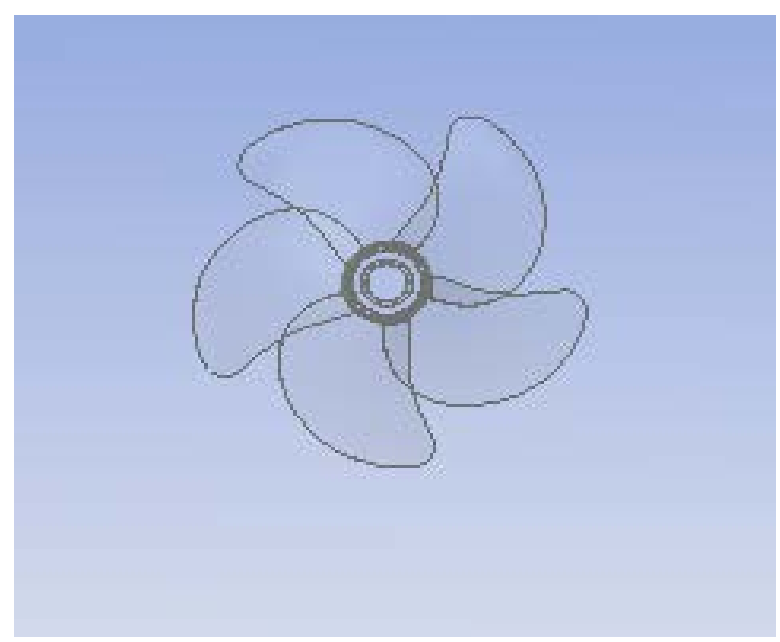

Fig.1. Boolean geometry in DesignModeler

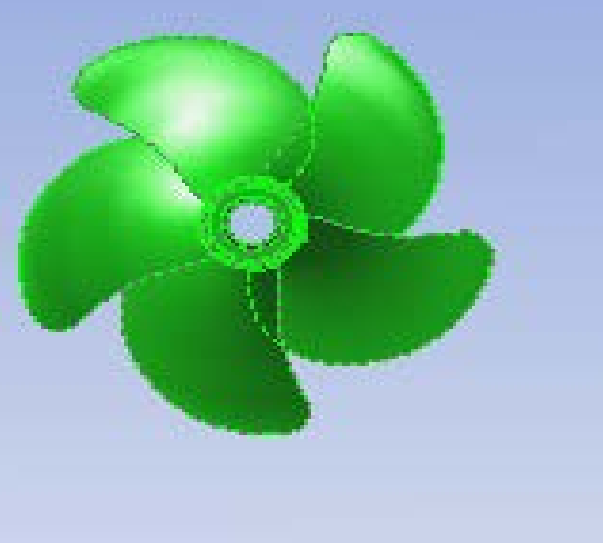

Fig.2. STP generated geometry for CFD test

Mesh generation is the practice in Software Ansys of generating a polygonal or polyhedral mesh that approximates a geometric fluid domain. The term "grid generation" is often used interchangeably. Typical uses are for rendering to a computer screen or for physical simulation such as finite element analysis or computational fluid dynamics and for simple problem solving.. 
"Mircea cel Batran" Naval Academy Scientific Bulletin, Volume XX - 2017 - Issue 2

Published by "Mircea cel Batran" Naval Academy Press, Romania /I The journal is indexed in: PROQUEST I DOAJ / DRJI / JOURNAL INDEX I I2OR / SCIENCE LIBRARY INDEX / Google Scholar / Crossref / Academic Keys I ROAD Open Access I OAJI / Academic Resources / Scientific Indexing Services / SCIPIO / JIFACTOR
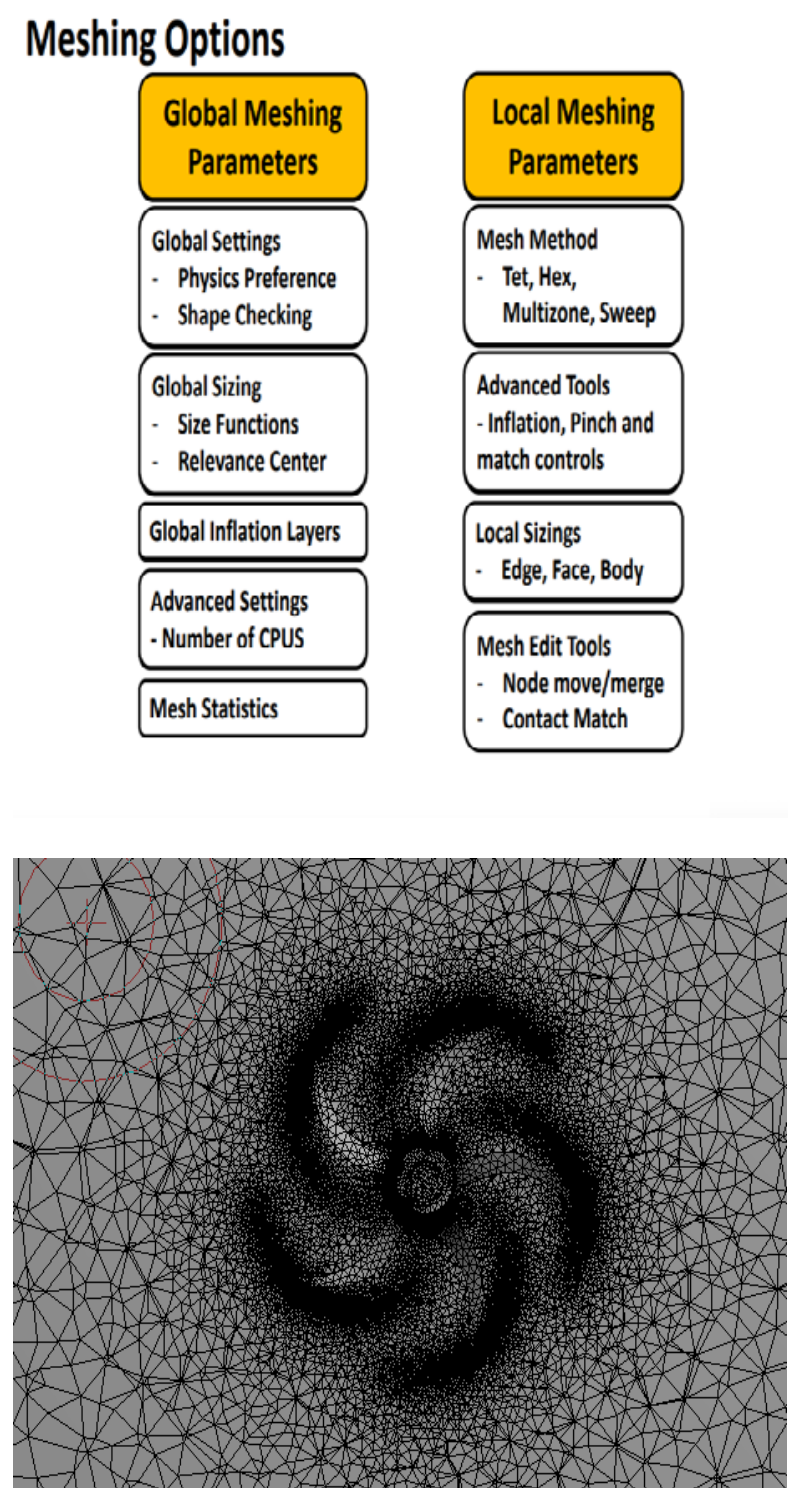

Fig.3. Mesh generation in Mesh Component for CFX

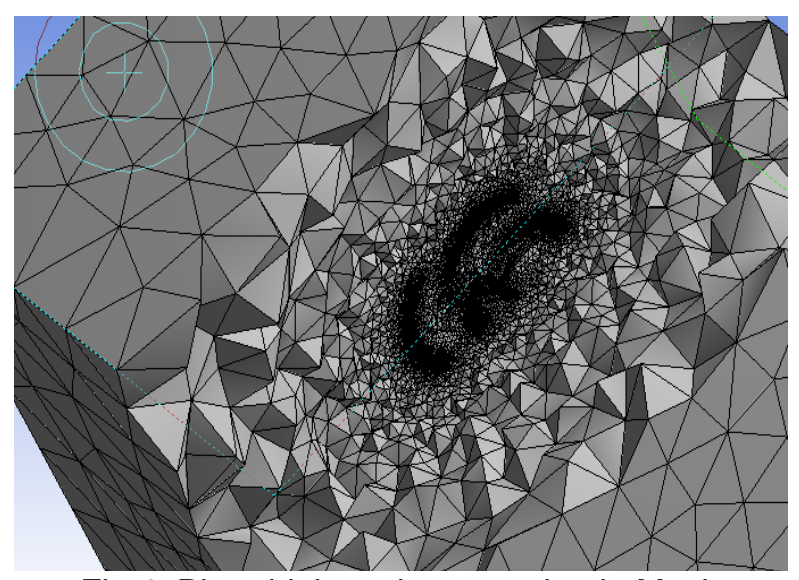

Fig.4. Piramidal mesh generation in Mesh Component for CFX
Meshing work flow: preparing geometry for simulation

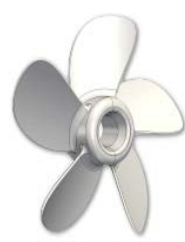

Simulation domain (geometry representation)

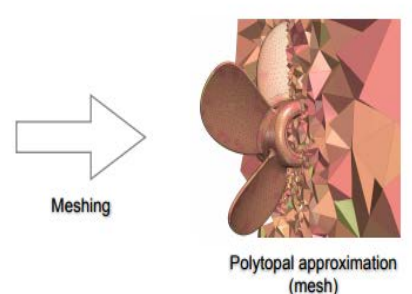

(mesh)
Fig.5. Preparing for CFD simulation

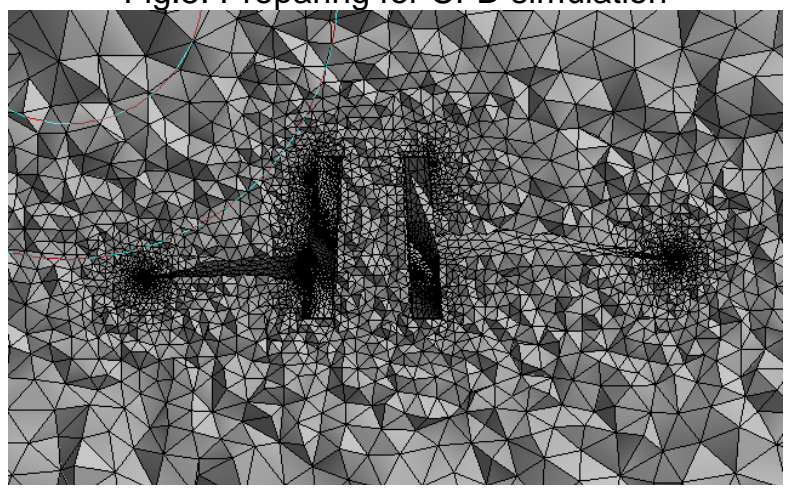

Fig.6. Vertical piramidal mesh generation in Mesh Component for CFX

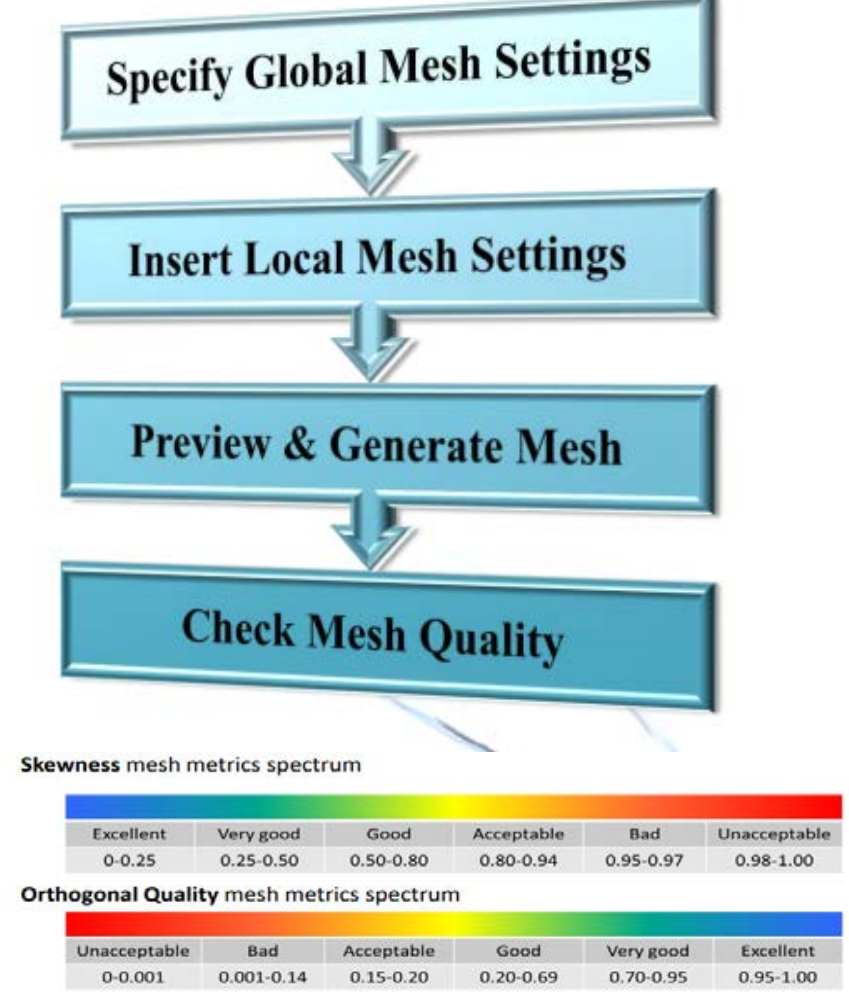

Fig.7. Mesh test for quality 
“Mircea cel Batran" Naval Academy Scientific Bulletin, Volume XX - 2017 - Issue 2

Published by "Mircea cel Batran" Naval Academy Press, Romania /l The journal is indexed in: PROQUEST I DOAJ / DRJI / JOURNAL INDEX / I2OR / SCIENCE LIBRARY INDEX / Google Scholar / Crossref / Academic Keys I ROAD Open Access / OAJI / Academic Resources / Scientific Indexing Services / SCIPIO / JIFACTOR

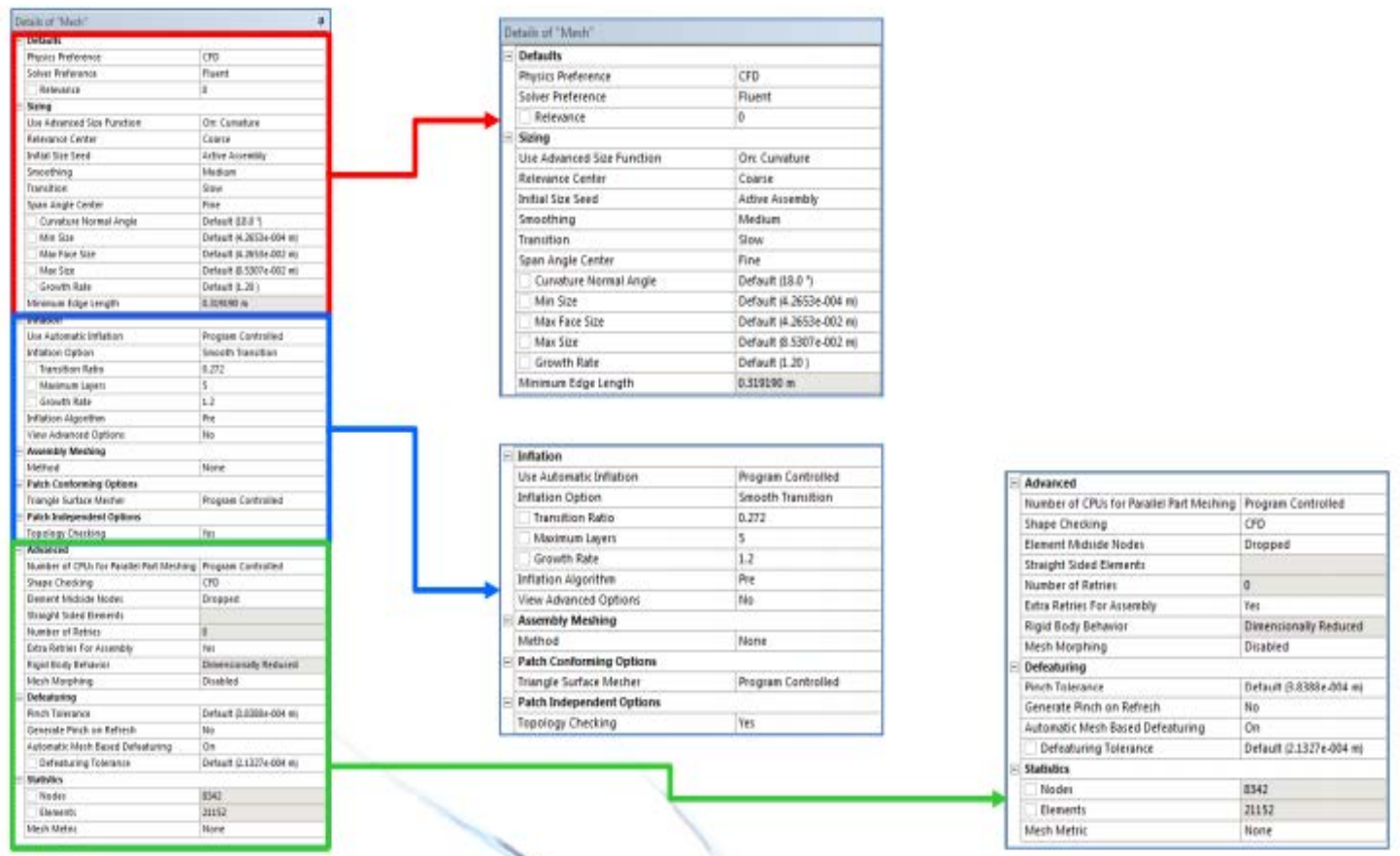

Global Mesh controls for optimal mesh in CFD

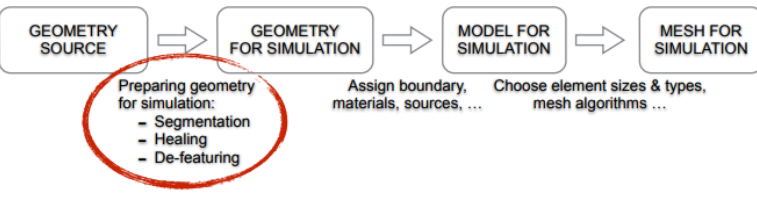

Fig.8. Simulation Process in Ansys Software

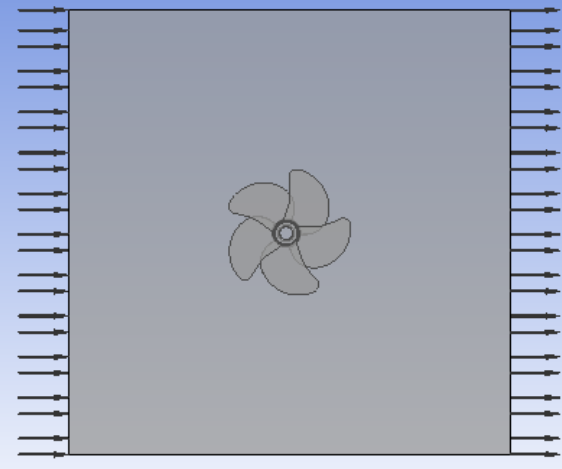

Fig.9. Cfx pre Settings for CFD test

Table 1. File Information for CFX

\begin{tabular}{|l|l|}
\hline Case & CFX \\
\hline $\begin{array}{l}\text { File } \\
\text { Path }\end{array}$ & $\begin{array}{l}\text { C:IUsers } \\
\text { XON- }\end{array}$ \\
\hline
\end{tabular}

\begin{tabular}{|l|l|}
\hline & $\begin{array}{l}\text { PC_7448_2lunsaved_project_filesldp0I } \\
\text { CFXICFXIFluid Flow CFX_001.res }\end{array}$ \\
\hline $\begin{array}{l}\text { File } \\
\text { Time }\end{array}$ & $12: 43: 34$ AM \\
\hline $\begin{array}{l}\text { File } \\
\text { Type }\end{array}$ & CFX5 \\
\hline $\begin{array}{l}\text { File } \\
\text { Versi } \\
\text { on }\end{array}$ & 14.5 \\
\hline
\end{tabular}

. Mesh Report

Table 2. Mesh Information for CFX

\begin{tabular}{|l|l|l|}
\hline Domain & Nodes & Elements \\
\hline Default Domain & 239622 & 1370393 \\
\hline
\end{tabular}

3. Physics Report

Table 3. Domain Physics for CFX

Domain - Default Domain 
"Mircea cel Batran" Naval Academy Scientific Bulletin, Volume XX - 2017 - Issue 2

Published by "Mircea cel Batran" Naval Academy Press, Romania // The journal is indexed in: PROQUEST I DOAJ / DRJI / JOURNAL INDEX / I2OR / SCIENCE LIBRARY INDEX / Google Scholar / Crossref / Academic Keys I ROAD Open Access / OAJI / Academic Resources / Scientific Indexing Services / SCIPIO / JIFACTOR

\begin{tabular}{|c|c|}
\hline Type & Fluid \\
\hline Location & B114 \\
\hline \multicolumn{2}{|l|}{ Materials } \\
\hline \multicolumn{2}{|l|}{ Air at $25 \mathrm{C}$} \\
\hline Fluid Definition & Material Library \\
\hline Morphology & Continuous Fluid \\
\hline \multicolumn{2}{|l|}{ Settings } \\
\hline Buoyancy Model & Non Buoyant \\
\hline Domain Motion & Stationary \\
\hline Reference Pressure & $1.0000 \mathrm{e}+00[\mathrm{~atm}]$ \\
\hline Heat Transfer Model & Isothermal \\
\hline Fluid Temperature & $2.5000 e+01[C]$ \\
\hline Turbulence Model & k epsilon \\
\hline Turbulent Wall Functions & Scalable \\
\hline
\end{tabular}

\begin{tabular}{|c|c|}
\hline $\begin{array}{l}\text { Flow } \\
\text { Regime }\end{array}$ & Subsonic \\
\hline $\begin{array}{l}\text { Mass And } \\
\text { Momentum }\end{array}$ & Normal Speed \\
\hline $\begin{array}{l}\text { Normal } \\
\text { Speed }\end{array}$ & $1.0000 \mathrm{e}+00\left[\mathrm{~m} \mathrm{~s}^{\wedge}-1\right]$ \\
\hline Turbulence & $\begin{array}{l}\text { Low Intensity and Eddy } \\
\text { Viscosity Ratio }\end{array}$ \\
\hline \multicolumn{2}{|c|}{ Boundary - Boundary 2} \\
\hline Type & OUTLET \\
\hline Location & outlet \\
\hline \multicolumn{2}{|l|}{ Settings } \\
\hline $\begin{array}{l}\text { Flow } \\
\text { Regime }\end{array}$ & Subsonic \\
\hline $\begin{array}{l}\text { Mass And } \\
\text { Momentum }\end{array}$ & $\begin{array}{l}\text { Average Static } \\
\text { Pressure }\end{array}$ \\
\hline $\begin{array}{l}\text { Pressure } \\
\text { Profile } \\
\text { Blend }\end{array}$ & $5.0000 e-02$ \\
\hline $\begin{array}{l}\text { Relative } \\
\text { Pressure }\end{array}$ & $0.0000 \mathrm{e}+00[\mathrm{~Pa}]$ \\
\hline $\begin{array}{l}\text { Pressure } \\
\text { Averaging }\end{array}$ & $\begin{array}{l}\text { Average Over Whole } \\
\text { Outlet }\end{array}$ \\
\hline \multicolumn{2}{|c|}{ Boundary - Default Domain Default } \\
\hline Type & WALL \\
\hline Location & $\begin{array}{l}F 115.114, F 116.114, \\
F 118.114, F 120.114, \\
F 121.114, F 122.114, \\
F 123.114, F 124.114, \\
F 125.114, F 126.114,\end{array}$ \\
\hline
\end{tabular}

Table 4. Boundary Physics for CFX

\begin{tabular}{|l|l|l|}
\hline Domain & \multicolumn{2}{|l|}{ Boundaries } \\
\hline \multirow{3}{*}{$\begin{array}{l}\text { Default } \\
\text { Domain }\end{array}$} & Boundary - Boundary 1 \\
\cline { 2 - 3 } & Type & INLET \\
\cline { 2 - 3 } & Location & inlet \\
\cline { 2 - 2 } & Settings \\
\hline
\end{tabular}


"Mircea cel Batran" Naval Academy Scientific Bulletin, Volume XX - 2017 - Issue 2

Published by "Mircea cel Batran" Naval Academy Press, Romania /I The journal is indexed in: PROQUEST I DOAJ / DRJI / JOURNAL INDEX / I2OR / SCIENCE LIBRARY INDEX / Google Scholar / Crossref / Academic Keys I ROAD Open Access / OAJI / Academic Resources / Scientific Indexing Services / SCIPIO / JIFACTOR

\begin{tabular}{|c|c|}
\hline & $\begin{array}{l}F 127.114, F 129.114, \\
F 131.114, F 132.114, \\
F 133.114, F 134.114, \\
F 135.114, F 136.114, \\
F 137.114, F 138.114, \\
F 139.114, F 140.114, \\
F 141.114, F 142.114, \\
F 143.114, F 144.114, \\
F 145.114, F 147.114, \\
F 148.114, F 149.114, \\
F 150.114, F 151.114, \\
F 153.114, F 154.114, \\
F 156.114, F 157.114,\end{array}$ \\
\hline \multicolumn{2}{|l|}{ Settings } \\
\hline $\begin{array}{l}\text { Mass And } \\
\text { Momentum }\end{array}$ & No Slip Wall \\
\hline $\begin{array}{l}\text { Wall } \\
\text { Roughness }\end{array}$ & Smooth Wall \\
\hline
\end{tabular}

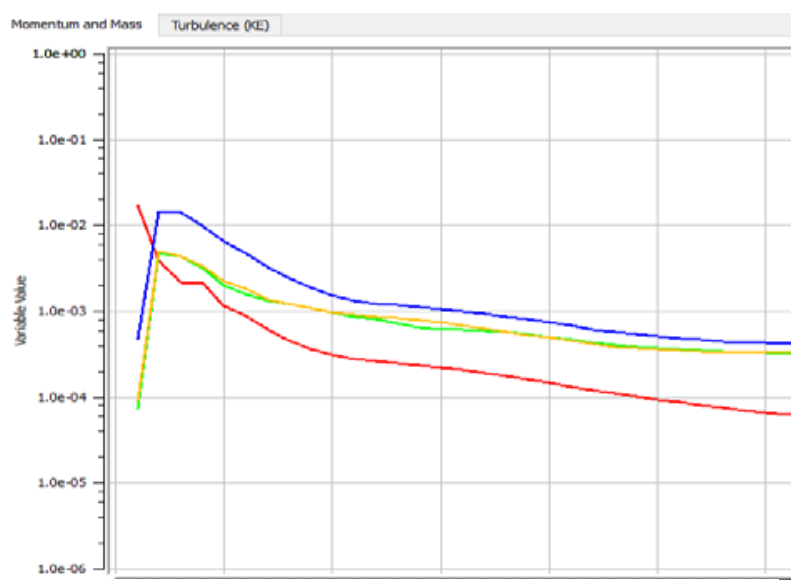

Fast Calculation for 10e-3 residuals in CFD test

Table 5. Boundary Flows for CFX - v1

\begin{tabular}{|l|l|l|l|l|l|}
\hline $\begin{array}{l}\text { Locat } \\
\text { ion }\end{array}$ & Type & $\begin{array}{l}\text { Mass } \\
\text { Flow }\end{array}$ & \multicolumn{3}{|l|}{ Momentum } \\
\cline { 4 - 6 } & & $\mathbf{X}$ & $\mathbf{Y}$ & $\mathbf{Z}$ \\
\hline $\begin{array}{l}\text { Boun } \\
\text { dary 1 }\end{array}$ & $\begin{array}{l}\text { Boun } \\
\text { dary }\end{array}$ & $\begin{array}{l}1.8159 \\
\mathrm{e}+02\end{array}$ & $\begin{array}{l}2.546 \\
2 \mathrm{e}-07\end{array}$ & $\begin{array}{l}- \\
1.8439 \\
\mathrm{e}+02\end{array}$ & $\begin{array}{l}9.355 \\
0 \mathrm{e}-07\end{array}$ \\
\hline $\begin{array}{l}\text { Boun } \\
\text { dary 2 }\end{array}$ & $\begin{array}{l}\text { Boun } \\
\text { dary }\end{array}$ & $\begin{array}{l}- \\
1.8159 \\
\mathrm{e}+02\end{array}$ & $\begin{array}{l}1.188 \\
8 \mathrm{e}-01\end{array}$ & $\begin{array}{l}1.8187 \\
\mathrm{e}+02\end{array}$ & $\begin{array}{l}4.600 \\
0 \mathrm{e}-03\end{array}$ \\
\hline Defau & Boun & 0.0000 & 1.218 & 2.5480 & - \\
\hline
\end{tabular}

\begin{tabular}{|l|l|l|l|l|l|}
\hline It & dary & $\mathrm{e}+00$ & $9 \mathrm{e}-01$ & $\mathrm{e}+00$ & 1.638 \\
Doma & & & & & $8 \mathrm{e}-03$ \\
in \\
$\begin{array}{l}\text { Defau } \\
\text { It }\end{array}$ & & & & & \\
\hline
\end{tabular}

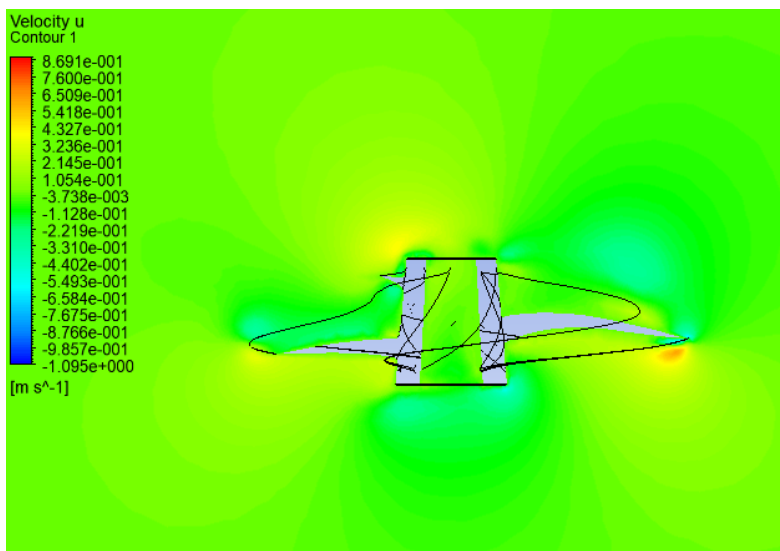

Fig.10. Velocity u contour on a 5 bladed propeller

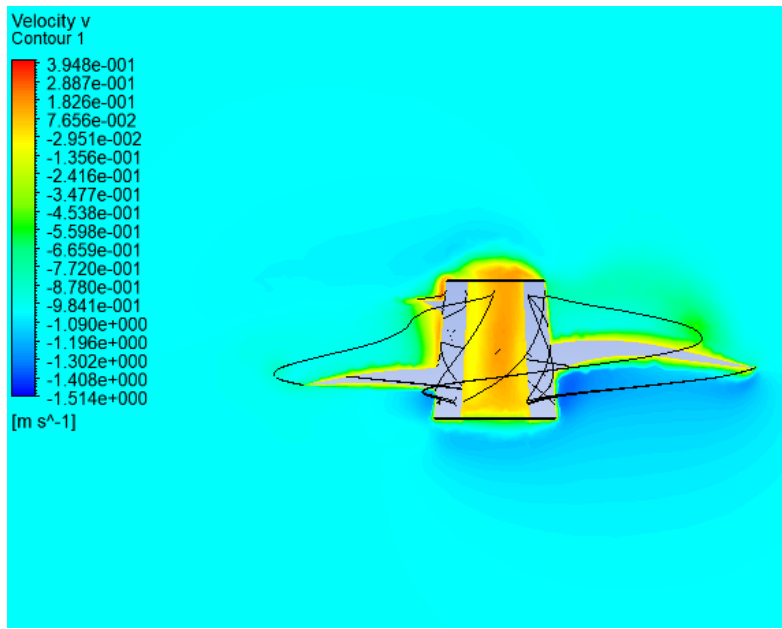

Fig.11. Velocity $v$ contour on a 5 bladed propeller

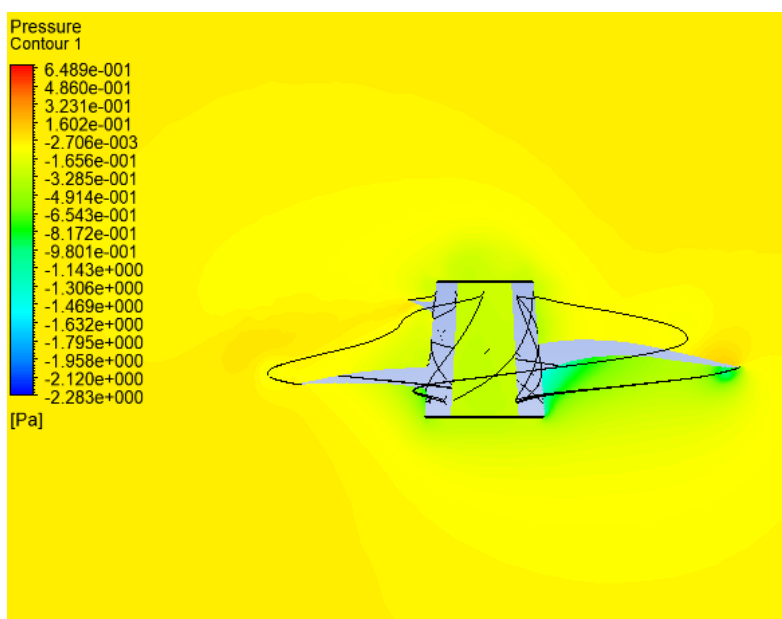

Fig.12. Pressure contour on a 5 bladed propeller 
"Mircea cel Batran" Naval Academy Scientific Bulletin, Volume XX - 2017 - Issue 2

Published by "Mircea cel Batran" Naval Academy Press, Romania // The journal is indexed in: PROQUEST I DOAJ / DRJI / JOURNAL INDEX / I2OR / SCIENCE LIBRARY INDEX / Google Scholar / Crossref / Academic Keys I ROAD Open Access / OAJI / Academic Resources / Scientific Indexing Services / SCIPIO / JIFACTOR

Table 6. Boundary Flows for CFX -v2

\begin{tabular}{|l|l|l|l|l|l|}
\hline \multirow{2}{*}{$\begin{array}{l}\text { Locat } \\
\text { ion }\end{array}$} & Type & $\begin{array}{l}\text { Mass } \\
\text { Flow }\end{array}$ & \multicolumn{3}{|l|}{ Momentum } \\
\cline { 4 - 6 } & & X & Y & Z \\
\hline $\begin{array}{l}\text { Boun } \\
\text { dary 1 }\end{array}$ & $\begin{array}{l}\text { Boun } \\
\text { dary }\end{array}$ & $\begin{array}{l}1.8159 \\
\mathrm{e}+02\end{array}$ & $\begin{array}{l}2.746 \\
2 \mathrm{e}-07\end{array}$ & $\begin{array}{l}- \\
1.8838 \\
\mathrm{e}+02\end{array}$ & $\begin{array}{l}9.256 \\
0 \mathrm{e}-07\end{array}$ \\
\hline $\begin{array}{l}\text { Boun } \\
\text { dary 2 }\end{array}$ & $\begin{array}{l}\text { Boun } \\
\text { dary }\end{array}$ & $\begin{array}{l}1.6159 \\
\mathrm{e}+02\end{array}$ & $\begin{array}{l}1.168 \\
8 \mathrm{e}-01\end{array}$ & $\begin{array}{l}1.8187 \\
\mathrm{e}+02\end{array}$ & $\begin{array}{l}4.600 \\
0 \mathrm{e}-03\end{array}$ \\
\hline $\begin{array}{l}\text { Defau } \\
\text { It } \\
\begin{array}{l}\text { Doma } \\
\text { in } \\
\text { Defau } \\
\text { It }\end{array}\end{array}$ & $\begin{array}{l}\text { Boun } \\
\text { dary }\end{array}$ & $\begin{array}{l}0.0000 \\
\mathrm{e}+00\end{array}$ & $\begin{array}{l}1.118 \\
9 \mathrm{e}-01\end{array}$ & $\begin{array}{l}2.1480 \\
\mathrm{e}+00\end{array}$ & $\begin{array}{l}1.638 \\
8 \mathrm{e}-03\end{array}$ \\
\hline
\end{tabular}

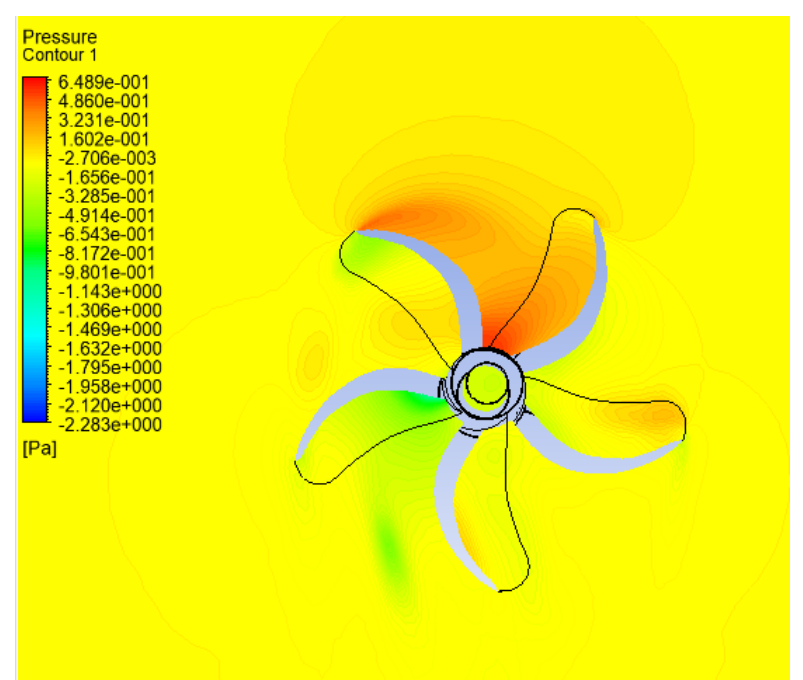

Fig.13. Pressure contour on a 5 bladed propeller

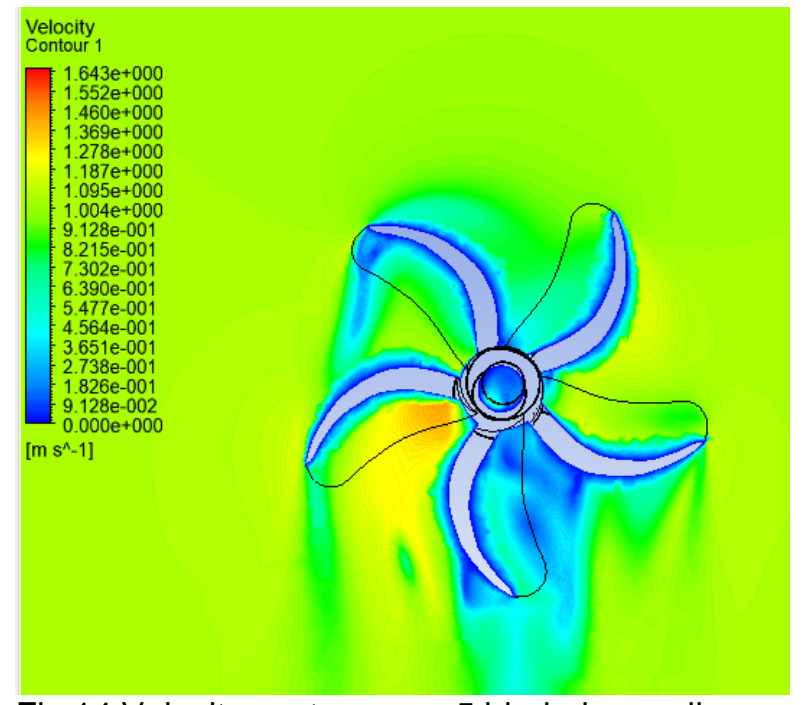

Fig.14 Velocity contour on a 5 bladed propeller
Table 7. Forces and Torques for CFX-v1

\begin{tabular}{|c|c|c|c|c|}
\hline Location & Type & $x$ & $Y$ & Z \\
\hline \multirow{6}{*}{$\begin{array}{l}\text { Default } \\
\text { Fluid } \\
\text { FluidInte } \\
\text { rface } \\
\text { Side } 1\end{array}$} & $\begin{array}{l}\text { Pressure } \\
\text { Force }\end{array}$ & $\begin{array}{l}0.0000 \\
e+00\end{array}$ & $\begin{array}{l}0.0000 \\
e+00\end{array}$ & $\begin{array}{l}0.0000 \\
e+00\end{array}$ \\
\hline & $\begin{array}{l}\text { Viscous } \\
\text { Force }\end{array}$ & $\begin{array}{l}0.0000 \\
e+00\end{array}$ & $\begin{array}{l}0.0000 \\
e+00\end{array}$ & $\begin{array}{l}0.0000 \\
e+00\end{array}$ \\
\hline & $\begin{array}{l}\text { Total } \\
\text { Force }\end{array}$ & $\begin{array}{l}0.0000 \\
e+00\end{array}$ & $\begin{array}{l}0.0000 \\
e+00\end{array}$ & $\begin{array}{l}0.0000 \\
e+00\end{array}$ \\
\hline & $\begin{array}{l}\text { Pressure } \\
\text { Torque }\end{array}$ & $\begin{array}{l}0.0000 \\
e+00\end{array}$ & $\begin{array}{l}0.0000 \\
e+00\end{array}$ & $\begin{array}{l}0.0000 \\
e+00\end{array}$ \\
\hline & $\begin{array}{l}\text { Viscous } \\
\text { Torque }\end{array}$ & $\begin{array}{l}0.0000 \\
e+00\end{array}$ & $\begin{array}{l}0.0000 \\
e+00\end{array}$ & $\begin{array}{l}0.0000 \\
e+00\end{array}$ \\
\hline & $\begin{array}{l}\text { Total } \\
\text { Torque }\end{array}$ & $\begin{array}{l}0.0000 \\
e+00\end{array}$ & $\begin{array}{l}0.0000 \\
e+00\end{array}$ & $\begin{array}{l}0.0000 \\
e+00\end{array}$ \\
\hline \multirow{6}{*}{$\begin{array}{l}\text { Default } \\
\text { Fluid } \\
\text { FluidInte } \\
\text { rface } \\
\text { Side } 11\end{array}$} & $\begin{array}{l}\text { Pressure } \\
\text { Force }\end{array}$ & $\begin{array}{l}0.0000 \\
e+00\end{array}$ & $\begin{array}{l}0.0000 \\
e+00\end{array}$ & $\begin{array}{l}0.0000 \\
e+00\end{array}$ \\
\hline & $\begin{array}{l}\text { Viscous } \\
\text { Force }\end{array}$ & $\begin{array}{l}0.0000 \\
e+00\end{array}$ & $\begin{array}{l}0.0000 \\
e+00\end{array}$ & $\begin{array}{l}0.0000 \\
e+00\end{array}$ \\
\hline & $\begin{array}{l}\text { Total } \\
\text { Force }\end{array}$ & $\begin{array}{l}0.0000 \\
e+00\end{array}$ & $\begin{array}{l}0.0000 \\
e+00\end{array}$ & $\begin{array}{l}0.0000 \\
e+00\end{array}$ \\
\hline & $\begin{array}{l}\text { PressureT } \\
\text { orque }\end{array}$ & $\begin{array}{l}0.0000 \\
e+00\end{array}$ & $\begin{array}{l}0.0000 \\
e+00\end{array}$ & $\begin{array}{l}0.0000 \\
e+00\end{array}$ \\
\hline & $\begin{array}{l}\text { ViscousTo } \\
\text { rque }\end{array}$ & $\begin{array}{l}0.0000 \\
e+00\end{array}$ & $\begin{array}{l}0.0000 \\
e+00\end{array}$ & $\begin{array}{l}0.0000 \\
e+00\end{array}$ \\
\hline & $\begin{array}{l}\text { Total } \\
\text { Torque }\end{array}$ & $\begin{array}{l}0.0000 \\
e+00\end{array}$ & $\begin{array}{l}0.0000 \\
e+00\end{array}$ & $\begin{array}{l}0.0000 \\
e+00\end{array}$ \\
\hline \multirow{6}{*}{$\begin{array}{l}\text { Propelle } \\
r\end{array}$} & $\begin{array}{l}\text { Pressure } \\
\text { Force }\end{array}$ & $\begin{array}{l}- \\
6.9048 \\
e+01\end{array}$ & $\begin{array}{l}- \\
6.3854 \\
e+02\end{array}$ & $\begin{array}{l}- \\
6.4703 \\
e+00\end{array}$ \\
\hline & $\begin{array}{l}\text { Viscous } \\
\text { Force }\end{array}$ & $\begin{array}{l}3.6559 \\
\text { e-02 }\end{array}$ & $\begin{array}{l}2.8645 \\
e+00\end{array}$ & $\begin{array}{l}2.6887 \\
\text { e-02 }\end{array}$ \\
\hline & $\begin{array}{l}\text { Total } \\
\text { Force }\end{array}$ & $\begin{array}{l}- \\
6.9011 \\
e+01\end{array}$ & $\begin{array}{l}- \\
6.3567 \\
e+02\end{array}$ & $\begin{array}{l}- \\
6.4434 \\
e+00\end{array}$ \\
\hline & $\begin{array}{l}\text { PressureT } \\
\text { orque }\end{array}$ & $\begin{array}{l}- \\
3.2999 \\
e+01\end{array}$ & $\begin{array}{l}1.0016 \\
e+02\end{array}$ & $\begin{array}{l}1.8079 \\
e+01\end{array}$ \\
\hline & $\begin{array}{l}\text { ViscousTo } \\
\text { rque }\end{array}$ & $\begin{array}{l}- \\
1.1762 \\
\text { e-02 }\end{array}$ & $\begin{array}{l}1.8317 \\
e+00\end{array}$ & $\begin{array}{l}- \\
2.1533 \\
\mathrm{e}-02\end{array}$ \\
\hline & $\begin{array}{l}\text { Total } \\
\text { Torque }\end{array}$ & $\begin{array}{l}- \\
3.2016 \\
e+01\end{array}$ & $\begin{array}{l}1.1199 \\
e+02\end{array}$ & $\begin{array}{l}1.8056 \\
e+01\end{array}$ \\
\hline Lateral & $\begin{array}{l}\text { Pressure } \\
\text { Force }\end{array}$ & $\begin{array}{l}- \\
1.7228 \\
e+02\end{array}$ & $\begin{array}{l}1.4969 \\
e+00\end{array}$ & $\begin{array}{l}1.1239 \\
e+02\end{array}$ \\
\hline
\end{tabular}


"Mircea cel Batran" Naval Academy Scientific Bulletin, Volume XX - 2017 - Issue 2

Published by "Mircea cel Batran" Naval Academy Press, Romania // The journal is indexed in: PROQUEST I DOAJ / DRJI / JOURNAL INDEX I I2OR / SCIENCE LIBRARY INDEX / Google Scholar / Crossref / Academic Keys I ROAD Open Access / OAJI / Academic Resources / Scientific Indexing Services / SCIPIO / JIFACTOR

\begin{tabular}{|c|c|c|c|}
\hline $\begin{array}{l}\text { Viscous } \\
\text { Force }\end{array}$ & $\begin{array}{l}- \\
4.4316 \\
e-04\end{array}$ & $\begin{array}{l}3.3752 \\
e-03\end{array}$ & $\begin{array}{l}1.1807 \\
\mathrm{e}-03\end{array}$ \\
\hline $\begin{array}{l}\text { Total } \\
\text { Force }\end{array}$ & $\begin{array}{l}- \\
1.3228 \\
e+02\end{array}$ & $\begin{array}{l}1.5006 \\
e+00\end{array}$ & $\begin{array}{l}1.9439 \\
e+02\end{array}$ \\
\hline $\begin{array}{l}\text { PressureT } \\
\text { orque }\end{array}$ & $\begin{array}{l}- \\
5.3516 \\
e+00\end{array}$ & $\begin{array}{l}1.2585 \\
e+01\end{array}$ & $\begin{array}{l}1.0824 \\
e+02\end{array}$ \\
\hline $\begin{array}{l}\text { ViscousTo } \\
\text { rque }\end{array}$ & $\begin{array}{l}2.2189 \\
\mathrm{e}-03\end{array}$ & $\begin{array}{l}9.2709 \\
\text { e-05 }\end{array}$ & $\begin{array}{l}1.8172 \\
\text { e-03 }\end{array}$ \\
\hline $\begin{array}{l}\text { Total } \\
\text { Torque }\end{array}$ & $\begin{array}{l}- \\
5.8486 \\
e+00\end{array}$ & $\begin{array}{l}1.1585 \\
\mathrm{e}+01\end{array}$ & $\begin{array}{l}1.0825 \\
e+02\end{array}$ \\
\hline
\end{tabular}

Table 8. Forces and Torques for CFX v2

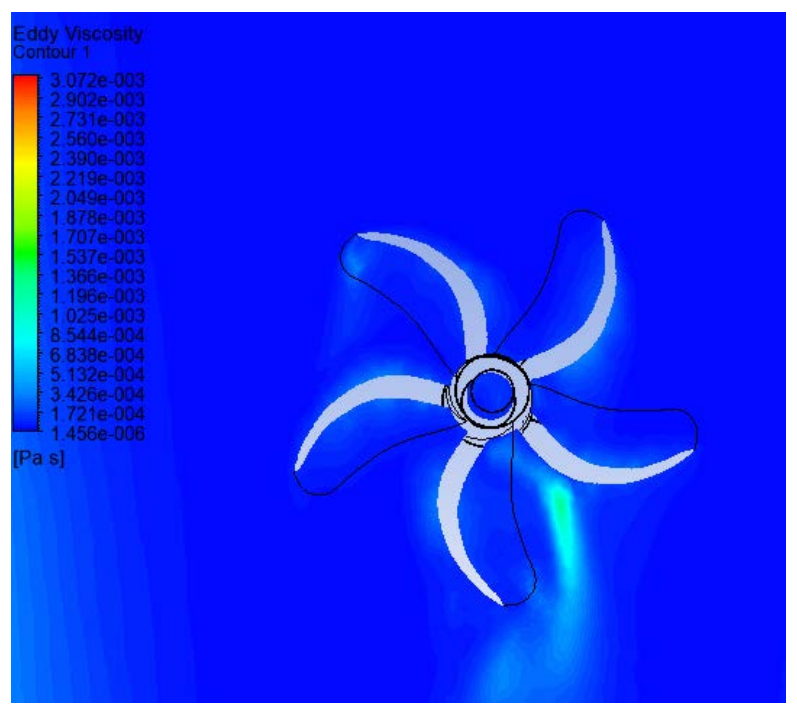

Fig.15. Eddy viscosity on CFD test

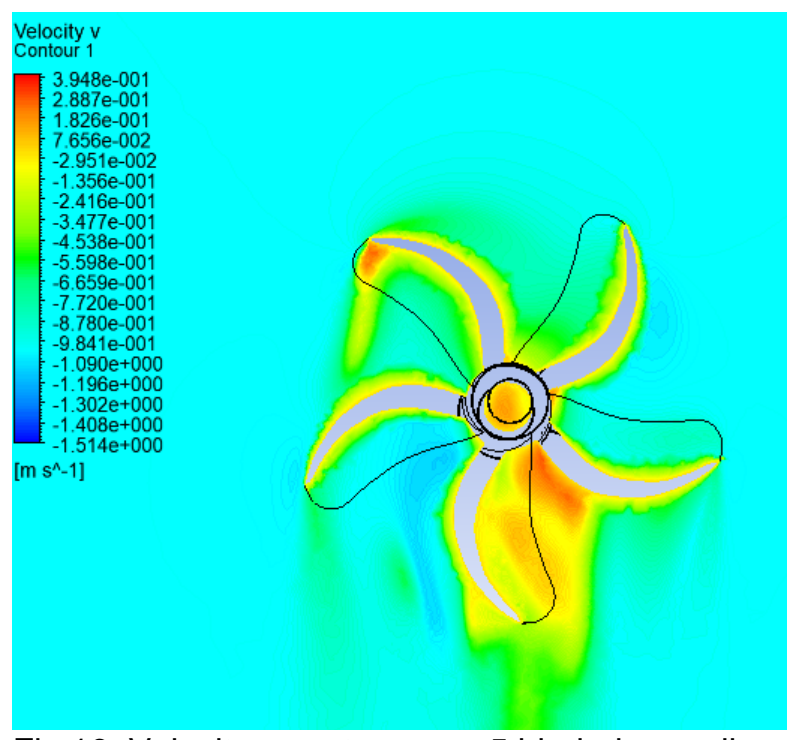

Fig.16. Velocity v contour on a 5 bladed propeller

\begin{tabular}{|c|c|c|c|c|}
\hline Location & Type & $x$ & $Y$ & Z \\
\hline \multirow{6}{*}{$\begin{array}{l}\text { Default } \\
\text { Fluid } \\
\text { FluidInte } \\
\text { rface } \\
\text { Side } 1\end{array}$} & $\begin{array}{l}\text { Pressure } \\
\text { Force }\end{array}$ & $\begin{array}{l}0.0000 \\
e+00\end{array}$ & $\begin{array}{l}0.0000 \\
e+00\end{array}$ & $\begin{array}{l}0.0000 \\
e+00\end{array}$ \\
\hline & $\begin{array}{l}\text { Viscous } \\
\text { Force }\end{array}$ & $\begin{array}{l}0.0000 \\
e+00\end{array}$ & $\begin{array}{l}0.0000 \\
e+00\end{array}$ & $\begin{array}{l}0.0000 \\
e+00\end{array}$ \\
\hline & $\begin{array}{l}\text { Total } \\
\text { Force }\end{array}$ & $\begin{array}{l}0.0000 \\
e+00\end{array}$ & $\begin{array}{l}0.0000 \\
e+00\end{array}$ & $\begin{array}{l}0.0000 \\
e+00\end{array}$ \\
\hline & $\begin{array}{l}\text { Pressure } \\
\text { Torque }\end{array}$ & $\begin{array}{l}0.0000 \\
e+00\end{array}$ & $\begin{array}{l}0.0000 \\
e+00\end{array}$ & $\begin{array}{l}0.0000 \\
e+00\end{array}$ \\
\hline & $\begin{array}{l}\text { Viscous } \\
\text { Torque }\end{array}$ & $\begin{array}{l}0.0000 \\
e+00\end{array}$ & $\begin{array}{l}0.0000 \\
e+00\end{array}$ & $\begin{array}{l}0.0000 \\
e+00\end{array}$ \\
\hline & $\begin{array}{l}\text { Total } \\
\text { Torque }\end{array}$ & $\begin{array}{l}0.0000 \\
e+00\end{array}$ & $\begin{array}{l}0.0000 \\
e+00\end{array}$ & $\begin{array}{l}0.0000 \\
e+00\end{array}$ \\
\hline \multirow{6}{*}{$\begin{array}{l}\text { Default } \\
\text { Fluid } \\
\text { FluidInte } \\
\text { rface } \\
\text { Side } 11\end{array}$} & $\begin{array}{l}\text { Pressure } \\
\text { Force }\end{array}$ & $\begin{array}{l}0.0000 \\
e+00\end{array}$ & $\begin{array}{l}0.0000 \\
e+00\end{array}$ & $\begin{array}{l}0.0000 \\
e+00\end{array}$ \\
\hline & $\begin{array}{l}\text { Viscous } \\
\text { Force }\end{array}$ & $\begin{array}{l}0.0000 \\
e+00\end{array}$ & $\begin{array}{l}0.0000 \\
e+00\end{array}$ & $\begin{array}{l}0.0000 \\
e+00\end{array}$ \\
\hline & $\begin{array}{l}\text { Total } \\
\text { Force }\end{array}$ & $\begin{array}{l}0.0000 \\
e+00\end{array}$ & $\begin{array}{l}0.0000 \\
e+00\end{array}$ & $\begin{array}{l}0.0000 \\
e+00\end{array}$ \\
\hline & $\begin{array}{l}\text { PressureT } \\
\text { orque }\end{array}$ & $\begin{array}{l}0.0000 \\
e+00\end{array}$ & $\begin{array}{l}0.0000 \\
e+00\end{array}$ & $\begin{array}{l}0.0000 \\
e+00\end{array}$ \\
\hline & $\begin{array}{l}\text { ViscousTo } \\
\text { rque }\end{array}$ & $\begin{array}{l}0.0000 \\
e+00\end{array}$ & $\begin{array}{l}0.0000 \\
e+00\end{array}$ & $\begin{array}{l}0.0000 \\
e+00\end{array}$ \\
\hline & $\begin{array}{l}\text { Total } \\
\text { Torque }\end{array}$ & $\begin{array}{l}0.0000 \\
e+00\end{array}$ & $\begin{array}{l}0.0000 \\
e+00\end{array}$ & $\begin{array}{l}0.0000 \\
e+00\end{array}$ \\
\hline \multirow{6}{*}{$\begin{array}{l}\text { Propelle } \\
r\end{array}$} & $\begin{array}{l}\text { Pressure } \\
\text { Force }\end{array}$ & $\begin{array}{l}- \\
6.9048 \\
e+01\end{array}$ & $\begin{array}{l}- \\
6.3854 \\
e+02\end{array}$ & $\begin{array}{l}- \\
6.4703 \\
e+00\end{array}$ \\
\hline & $\begin{array}{l}\text { Viscous } \\
\text { Force }\end{array}$ & $\begin{array}{l}3.6559 \\
\text { e-02 }\end{array}$ & $\begin{array}{l}2.8645 \\
e+00\end{array}$ & $\begin{array}{l}2.6887 \\
\text { e-02 }\end{array}$ \\
\hline & $\begin{array}{l}\text { Total } \\
\text { Force }\end{array}$ & $\begin{array}{l}- \\
6.9011 \\
e+01\end{array}$ & $\begin{array}{l}- \\
6.3567 \\
e+02\end{array}$ & $\begin{array}{l}- \\
6.4434 \\
e+00\end{array}$ \\
\hline & $\begin{array}{l}\text { PressureT } \\
\text { orque }\end{array}$ & $\begin{array}{l}- \\
3.2999 \\
e+01\end{array}$ & $\begin{array}{l}1.0016 \\
e+02\end{array}$ & $\begin{array}{l}1.8079 \\
e+01\end{array}$ \\
\hline & $\begin{array}{l}\text { ViscousTo } \\
\text { rque }\end{array}$ & $\begin{array}{l}- \\
1.1762 \\
\text { e-02 }\end{array}$ & $\begin{array}{l}1.8317 \\
e+00\end{array}$ & $\begin{array}{l}- \\
2.1533 \\
\mathrm{e}-02\end{array}$ \\
\hline & $\begin{array}{l}\text { Total } \\
\text { Torque }\end{array}$ & $\begin{array}{l}- \\
3.2016 \\
e+01\end{array}$ & $\begin{array}{l}1.1199 \\
e+02\end{array}$ & $\begin{array}{l}1.8056 \\
e+01\end{array}$ \\
\hline Lateral & $\begin{array}{l}\text { Pressure } \\
\text { Force }\end{array}$ & $\begin{array}{l}- \\
1.7228 \\
e+02\end{array}$ & $\begin{array}{l}1.4969 \\
e+00\end{array}$ & $\begin{array}{l}1.1239 \\
e+02\end{array}$ \\
\hline
\end{tabular}


"Mircea cel Batran" Naval Academy Scientific Bulletin, Volume XX - 2017 - Issue 2

Published by "Mircea cel Batran" Naval Academy Press, Romania /l The journal is indexed in: PROQUEST I

DOAJ / DRJI / JOURNAL INDEX I I2OR / SCIENCE LIBRARY INDEX / Google Scholar / Crossref / Academic Keys I ROAD Open Access / OAJI / Academic Resources / Scientific Indexing Services / SCIPIO / JIFACTOR

\begin{tabular}{|l|l|l|l|l|}
\hline \begin{tabular}{|l} 
Viscous \\
Force
\end{tabular} & $\begin{array}{l}\text { 4.4316 } \\
\mathrm{e}-04\end{array}$ & $\begin{array}{l}3.3752 \\
\mathrm{e}-03\end{array}$ & $\begin{array}{l}1.1807 \\
\mathrm{e}-03\end{array}$ \\
\cline { 2 - 5 } $\begin{array}{l}\text { Total } \\
\text { Force }\end{array}$ & $\begin{array}{l}\text { 1.3228 } \\
\mathrm{e}+02\end{array}$ & $\begin{array}{l}1.5006 \\
\mathrm{e}+00\end{array}$ & $\begin{array}{l}1.9439 \\
\mathrm{e}+02\end{array}$ \\
\cline { 2 - 5 } & $\begin{array}{l}\text { PressureT } \\
\text { orque }\end{array}$ & $\begin{array}{l}5.3516 \\
\mathrm{e}+00\end{array}$ & $\begin{array}{l}1.2585 \\
\mathrm{e}+01\end{array}$ & $\begin{array}{l}1.0824 \\
\mathrm{e}+02\end{array}$ \\
\hline $\begin{array}{l}\text { ViscousTo } \\
\text { rque }\end{array}$ & $\begin{array}{l}2.2189 \\
\mathrm{e}-03\end{array}$ & $\begin{array}{l}9.2709 \\
\mathrm{e}-05\end{array}$ & $\begin{array}{l}1.8172 \\
\mathrm{e}-03\end{array}$ \\
\hline & $\begin{array}{l}\text { Total } \\
\text { Torque }\end{array}$ & $\begin{array}{l}5.8486 \\
\mathrm{e}+00\end{array}$ & $\begin{array}{l}1.1585 \\
\mathrm{e}+01\end{array}$ & $\begin{array}{l}1.0825 \\
\mathrm{e}+02\end{array}$ \\
\hline
\end{tabular}

\section{CONCLUSION}

The Ansys software presented all data results from physical data to related calculations related to geometry, mesh and mesh quality in order to give a large vision on lateral simulation on a propeller. The CFX component was used to generate the characteristic velocity, pressure and turbulence ratios for the studied fluid field. Any propeller model can be verified in such an analysis at the design stage to understand the phenomena associated with propellers.

Velocities presented in contours are one of the most useful results in calculating various propeller related issues.

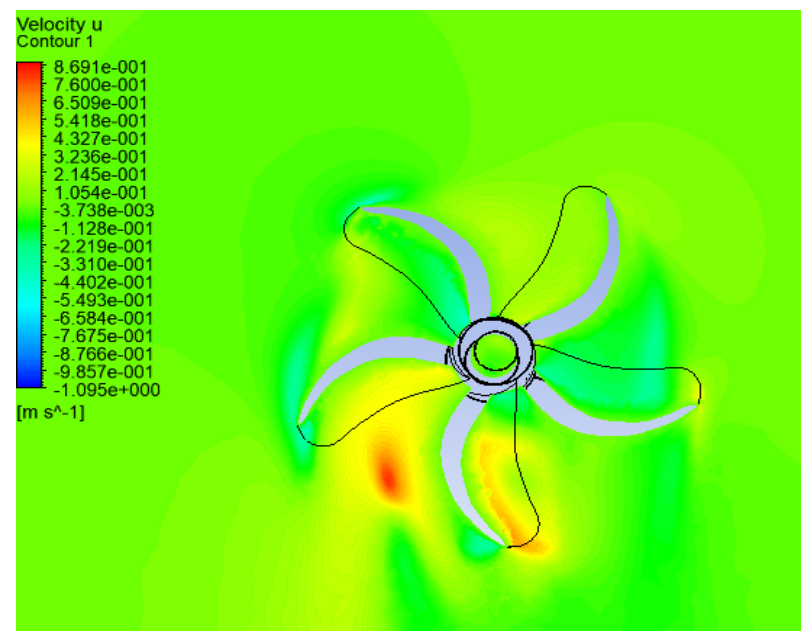

Fig.17. Velocity u contour on a 5 bladed propeller

\section{Bibliography}

[1] www.ansys.com

[2] Domnisoru L, Găvan E, POPOVICI O - Analiza structurilor navale prin metoda elementului finit, Editura Didactica si Pedagogica, Bucuresti 2005, ISBN $973-30$ - $1075-8$

[3] Călimănescu I., Stan L. C., Computer fluid dynamics (CFD)study of a micro annular gear pump, Atom 2016, Conference Paper.

[4] Stan L. Călimănescu I.,. C.A New innovative turbocharger concept numerically tested and optimised with CFD, 2016, Conference Paper.

[5]http://web.mit.edu/xeviroca/www/rocaMeshForSim.pdf

[6]https://www.padtinc.com/blog/wp-content/uploads/2017/04/Advanced-Techniques-in-ANSYS-

Meshing Blog.pdf

[7]https://www.ozeninc.com/wp-content/uploads/2014/11/MESHING WORKSHOP 2014.pdf 